\title{
Implementation of Adaptive Control Algorithm to Overcome the Traffic Congestion Problems of Karachi
}

\author{
${ }^{1}$ Aneel Ahmed, ${ }^{2}$ Faraz Junejo \\ ${ }^{1,2}$ Shaheed Zulfiqar Ali Bhutto Institute of Science and Technology, Karachi Pakistan \\ ${ }^{1}$ aneel $\mathrm{szabist} . \mathrm{edu} \cdot \mathrm{pk}$ \\ ${ }^{2}$ Faraz.junejo@szabist.edu.pk
}

\begin{abstract}
Traffic controlling and management is a severe issue of urban cities as well as on high ways in developing countries like South Asian countries but here particularly, in Pakistan. The traffic congestion problem is becoming more severe with time. The main reason behind all this is a drastic increase in number of running vehicles and no proper road infrastructure. Not only, the traffic congestion is a problem, whereas, the other problems are also associated with this issue are air pollution, wastage of resources like fuel, energy, time etc. are the foremost anomalies faced by every human being of third world countries. It is also one of the major reasons of global warming and exploitation of natural life system. Currently, in Pakistan traffic controlling is done through time based traffic control signal system normally.
\end{abstract}

In this paper, we have proposed a system which dynamically controls and manage the road traffic through image processing on real time and will take the decision on actual grounds for traffic routes and on traffic signal timing. In this system, we utilize the latest technologies of image processing which collects, organize and transmits the information to existing system to incorporate the new real time data for traffic timing control. It will save the people from road accidents and unnecessary wastage of fuel resources and more importantly make the life little bit more relax then others.

Keywords-Smart system; Image processing; Traffic signal, Adaptive algorithm.

\section{INTRODUCTION}

In $21^{\text {st }}$ century, there are number of studies that argues the role of technology in human life, constitution and identity [1-2]. These studies, most of the time concluded that humans have always shaped themselves utilizing several technological tools and artefacts. Let's think of MRI scan or a microscope; these gadgets became a compulsory part for medical investigation and diagnosis [3]. All the medication, treatment or even diet are planned on its results/reports. This means that technology occupies the human nature to much greater extent than it could be seen. These technological developments not only made human life easier and comfortable but luxurious as well [4-5].

However; in-spite of such modernization, there are some sectors of human life in which no major breakdown research has been done to give humans relief. With over 7.6 billion humans and 1.2 billion vehicles on road, the traffic congestion has become a part of everyday routine. Every day, regardless of their country and state, billions of peoples got stuck in traffic that not only cause their precious time but fuel as well. A major breakthrough needs to be done in the field of transportation that could address the following issue and make human life much more comfortable than before. As per the article published in The Forbes, only in America, the traffic congestion causes national economy a loss of \$ 124 billion in 2013 that has been forecasted to increase to $\$ 186$ billion by 2030 [6-8].

On the other hand, INRIX report also suggested that with the current rate of increase in population [9], by 2030 the situation of traffic shall become even worse that it is now. The united nations has also mentioned the importance of controlling the traffic congestion problem and put it as a target to 2030 global UN goals [10], "By 2030, provide access to safe, affordable, accessible and sustainable transport systems for all, improving road safety, notably by expanding public transport, with special attention to the needs of those in vulnerable situations, women, children, persons with disabilities and older persons".

In order to fight this global problem, some major work has to be done. The number of conceptual theories has been put forward that could solve the stated problem, some of them are given below:

1) Adaptive traffic signal

2) Autonomous vehicle technology

3) Replacing vehicle with drones 
From these stated designs currently, adaptive traffic signal sounds more realistic [11]. Adaptive traffic signals could solve this global traffic problem to wider extent while making changes to existing infrastructure to lesser extent. This paper focuses on the designing and implementation of Adaptive traffic signal in Karachi, 3-Talwar.

\section{A. Adaptive Traffic Signal:}

Currently installed traffic signals utilizes pre-programmed schedule of switching lights as per collected data. This data has been provided through constant monitoring of signal for finite period of time identifying the rush hours, slow hours and moderate hours. They also take into account the data of particular lane out of several so that manually time of single lane could be manipulated and fed in pre-programmed system. However, this pre-programming doesn't takes into account of such scenario like holiday, Election Day or strike. Adaptive traffic signal technology could adjust the timing of red, green and yellow lights as per current traffic patterns. This revolution could make system smart enough to make decision on its own while easing traffic congestion. The main benefits of these signals over conventional one's are:

- Improvement of traffic flow

- Faster responses to current traffic conditions

- Cutting costs

\section{a) Improvement of traffic flow:}

The problem with current traffic signals are that most of the time drivers have to waste their time staring at red light so that they could move, in spite of the fact that lane is clear. Or in other case, the lane got so congested that you should wait for some time before moving ahead but the traffic showed green and you left with no other option to start you vehicle and got stuck in there too. The idea is to make these signal intelligent enough to adapt real time conditions and make decisions accordingly [12].

The proposed technology can do exactly this. The system composed of various strategically placed sensors that extracts data on real time and send it to processor to process it and make decision accordingly. The sensor could be a well-placed camera that continuously takes pictures of the lanes and then send it to processor, processor processes it; applying different image processing techniques and makes decision as per the sett criteria. This could help in improving the service that locals or foreigner experience on roads and highways. Lesser the unnecessary delays, the faster and smoother is the traffic flow.

\section{b) Faster Responses to current traffic conditions:}

Currently, signal time has been altered by manually collected data which doesn't have any place to accommodate variables and unpredictable traffic demands. Due to these variables, drivers got stuck in traffic producing complaints and harmful safety. These complaints works as a form of feedback that help officials to twitch signal's timing. However, if there is no complaint made, than citizen may have to suffer from it for indefinite amount of time; whereas, adaptive signals could address these variables then and there; depending upon its working algorithm [13].

\section{c) Cutting costs:}

According to the study conducted by Texas transportation institute, around $\$ 87.2$ billion annually has been wasted in fuel and lost productivity due to traffic congestion. With indirectly translates to $\$ 750$ per head per citizen [14]. Currently used traffic signals causes a substantial cost to business and citizens. As per the stats they accounts for more than $10 \%$ of all traffic delays that causes excess fuel consumption and decreased business productivity. With adaptive signal control, all of the above mentioned cost is saved to greater extent intelligently. The data has been updated automatically. Most importantly, for citizen the signal timing updates are made as per the situation occurrence.

\section{SYSTEM INTEGRATION AND METHODOLOGY}

This study is proposing an integrated system that will help in addressing this global problem using digital image processing technique. Till now, most of the installed adaptive traffic signal utilizes transducers like IR or strain gauge to detect the presence of vehicle. However, this system will detect the presence using a camera that shall be installed alongside the traffic signal poles at $8-10 \mathrm{~m}$ of height above the ground levels. This will help the system to capture wide images on the basis of which suitable algorithm could be implemented.

As the raw images consist of several pixels, those pixels will be processed as per the specified criteria to generate desired results. The system will take a reference of an empty road picture that will be fed into it by user. The system will then compare every captured image with the default one and pursue a comparative analysis at the cost of their grey levels.

Each captured image will be distributed in three regions low, medium and high. These regions will be determined using the density function that will count the number of grey pixels in the image and compare it with the default one. Further some cases has been defined that will control the time of red, yellow and green light based on the detected region. The detailed process flow of the system is shown in Figure 1. 


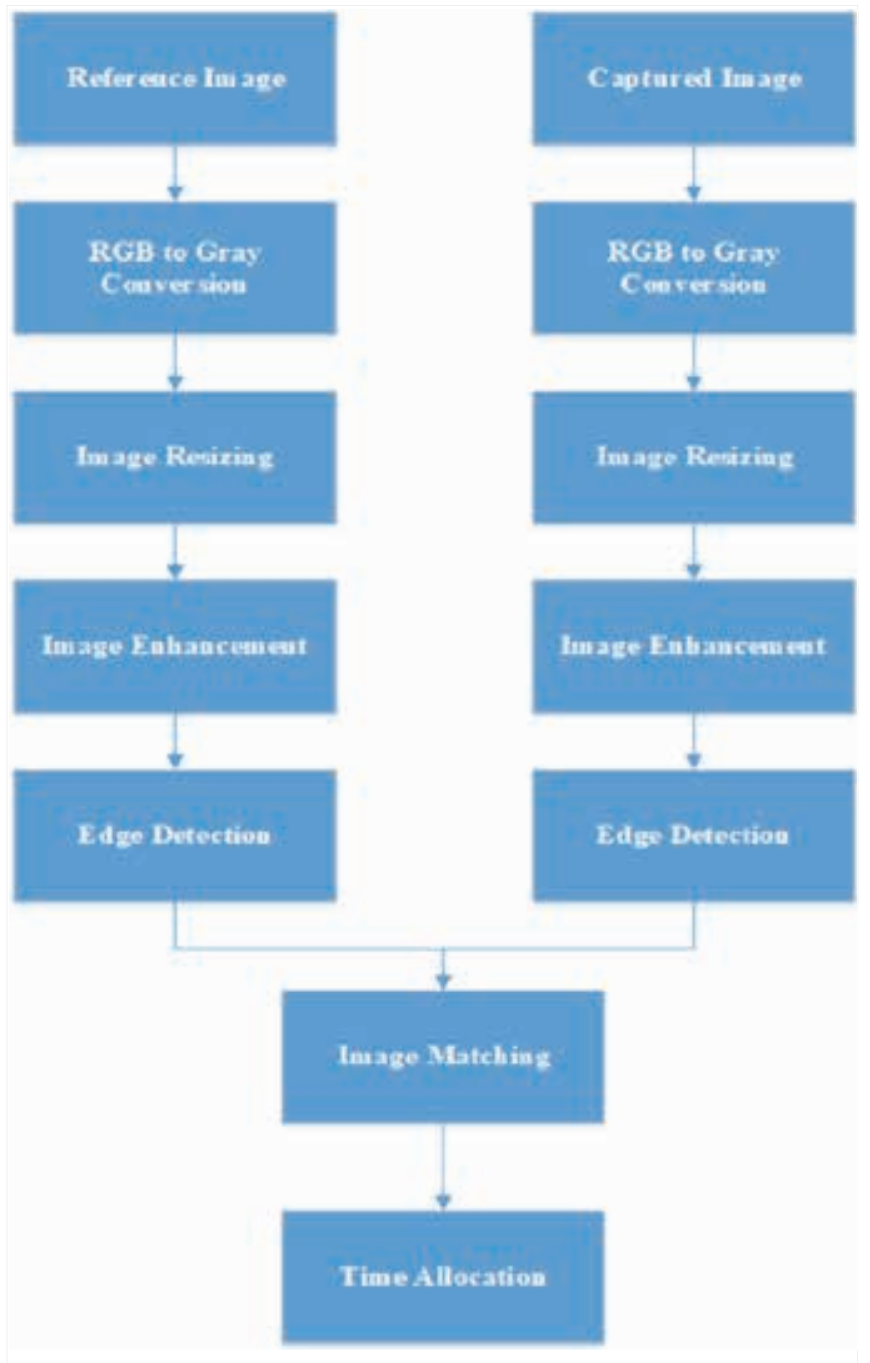

Fig. (1). System description of traffic signal algorithm

The objective of this proposed study is to make the existing system more $\mathrm{n}$ more efficient while utilizing the optimum existing resources by means of automating the traffic light system on the basis of density of traffic. This proposed system is solely based on image processing technique. As previously discussed that system has been design to be implemented on 3-Talwar, Karachi. The 3-Talwar signal is among one of the busiest signals in Karachi, where as per the statistics, 40-50 thousand cars passes daily.

Keeping in mind such a huge number, one can predict that what could be the condition of traffic there in rush hours. It's a four lane road consisting of 4 main traffic signals on a busy day, the signal looks like as shown in Figure 2.

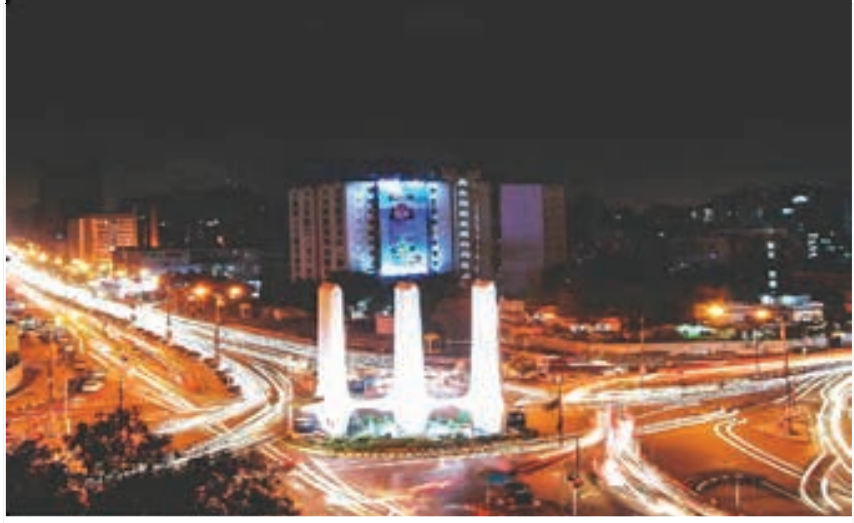

Fig. (2). 3-Talwar, busy day

Whereas, on slow days zero number of cars passing the signal could be seen in Figure 3.

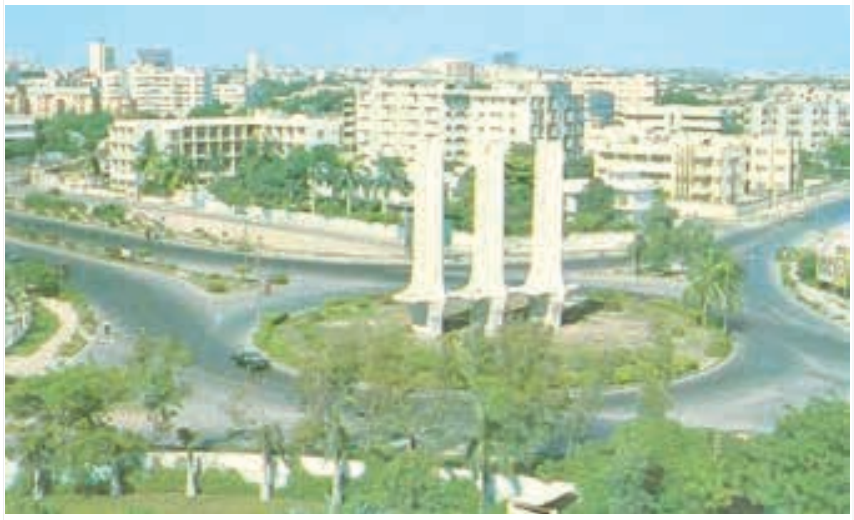

Fig. (3). 3-Talwar, slow day

Each side of traffic signal has assigned an initial timing of 10 seconds for green light and 5 seconds for yellow light and 60 seconds for red light and signal always follows the clockwise direction of signal shifting. Each traffic signal is mounted on an iron road of around 8-10m (approx. 24-30 ft.) and beside that a night vision high definition camera is mounted on each side of the traffic signal. The regions has been distributed as from 21-40 ft. it counts as low level density of vehicle, $41-60 \mathrm{ft}$. it count as medium level density of vehicles and 61-90 ft. it count as high level density of vehicles. Following these dimensions of regions the algorithm is designed in a way to follow different scenarios.

Initially it will start green for ten seconds and simultaneously it will also starts capturing images for 5 seconds, after acquiring images, the images are forwarded to microprocessor for further decision making, Let's think about the 1st scenario in which after initiating up to the medium level of density or less, the signal will indicate green light for 10 seconds and turns to red right after. As previously 
mentioned the signals will rotate turns in clockwise direction; therefore, it will jump on the other signal on which same cycle will be implemented.

In the Second scenario, if the vehicles are continuously reaching the high density region then at the start of the green light signal in first five seconds it will capture the image and send it to microprocessor for processing and microcontroller according to the algorithm resets the timing of the signal from 2 to 12 or from 0 to 10 more seconds this kind of loop will occur twice at max if same scenario exists otherwise it runs on initial timings and gives the turn to next.

All programming is done on MatLab environment in terms of modeling and simulation of this proposed system. Here we are using Otsu's Thresholding Approach instead of Global Thresholding. Because Global Thresholding is only be feasible when we have the well distributed histogram of our data set of images. A foremost limitation in Global Thresholding is that changes in illumination across the image and its background, which may cause some parts to be brighter in Light and some parts darker in in darkness, in that manner it is nothing to do with the objects in the image. It simply divides the group of pixels of an image into two greys levels and then computes the average of these threshold and these steps repeats until it reached to less than our predefined value of thresholding.

In contrast to it Otsu's Thresholding is more suitable in our case of study in which we have moving objects as well stationary [15]. Otsu's thresholding technique caters the strong illumination gradient, variance of the grey levels of an object and background as well as the inadequate contrast. In grey level of histogram we divide them into two groups and then find the threshold between them when the variance of both groups is maximum. This method provides good results even when the histogram of a grey level image does not have two obvious peaks using Equation 1. The detailed working of Otsu's algorithm is shown in in Figure 4.

PDF Gaussian $=\frac{1}{\sqrt{2 \pi} \sigma} \mathbf{e}^{\frac{(\mathrm{g}-\mu)^{2}}{2 \sigma^{2}}}$

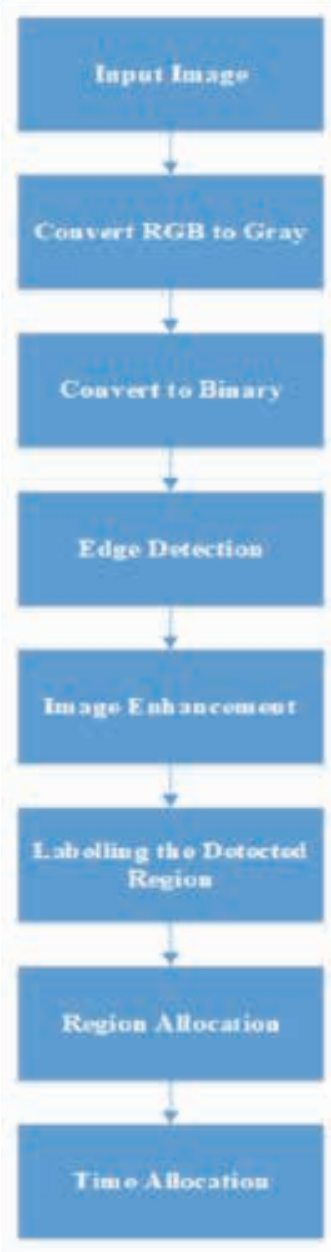

Fig. (4). Otsu's Algorithm

1. Firstly determine the maximum and minimum grey levels values of an image.

2. Then set the minimum grey level value as the initial threshold value as reference (To), based on this value of threshold, all the values of pixels of an image are divided into two groups, the values of grey level lesser or equal to the initial set threshold is represented by Co and pixel values of grey level which are higher to the initial set threshold value is represented by $\mathrm{C} 1$.

3. When are symbols of pixel numbers and average value of grey level of groups Co \& C1 respectively. Then the average grey level value of all the pixels can be calculated using Equation 2. 
4. Now we have to find the variance for the two groups of pixels in order to know how much data set of images are scattered from each other or form mean value of grey level. The variance is calculated using Equation 3.

$$
\sigma^{2}\left(T_{0}\right)=\left\{N_{0}\left(\mu_{0}-M_{T}\right)^{2}+N_{1}\left(\mu_{1}-M_{T}\right)^{2}\right\}
$$

5. In last we require the threshold value which is corresponds to maximum value of variance.

The combination of Otsu's algorithm with our system can be shown in Figure 5 below.

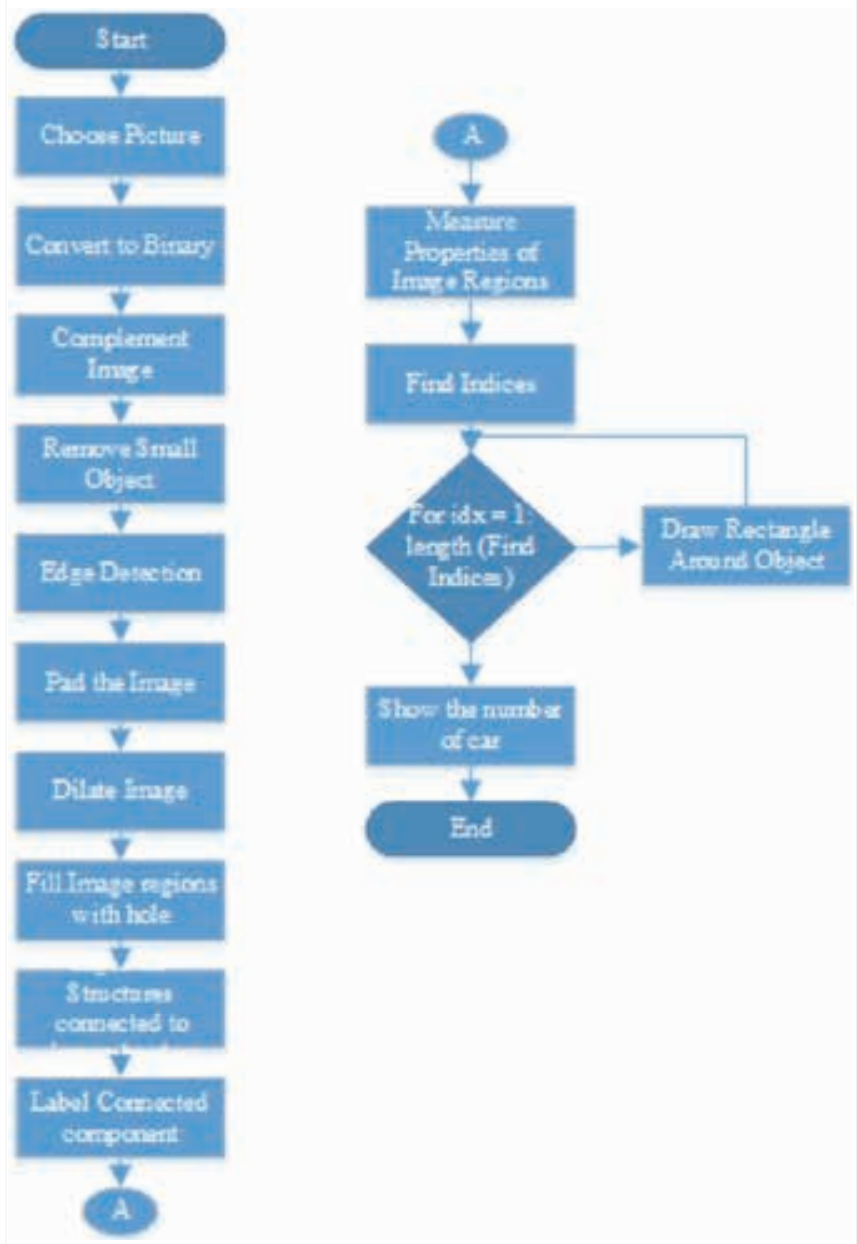

Fig. (5). Otsu's Algorithm implemented on our system

\section{ANALYSIS AND RESULTS}

On the basis of stated algorithm, the deep analysis of system has been shown in this section. With help of pictures each and every step of algorithm will be discussed. As discussed, for the system to work properly we have to fed reference image of an empty road, so that pixel density could be compared. Through this only we could identify the regions i.e. low, medium and high. The reference image of the system is shown in Figure 6.

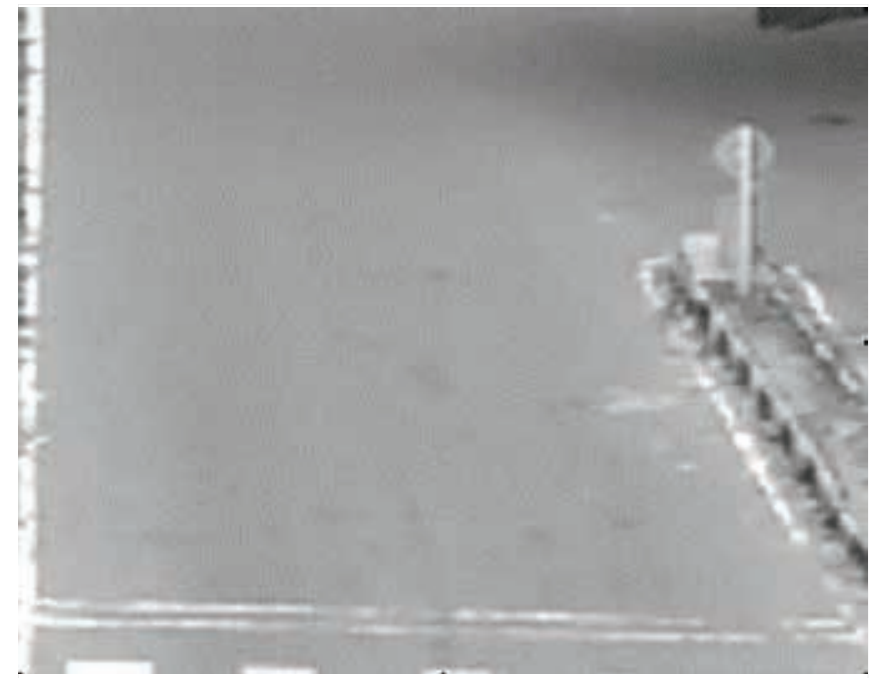

Fig. (6). Reference image

One image out of various is shown for analysis in Figure 7.

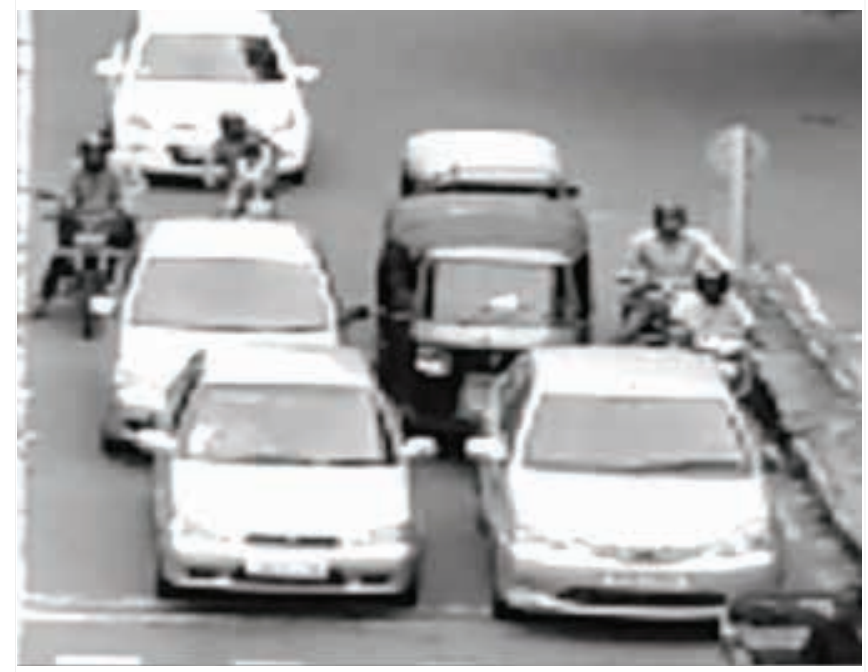

Fig. (7). Input image

The system will first convert the input image in grayscale, through this grayscale image the pixel density will be compared. Moving forward, the grey image will be compared to the reference one and then gets converted into inverted image as per our designed algorithm i.e. shown in Figure 8. For this purpose, we used an edge detection technique of canny operator on real time images for segmentation purpose. The important attributes of an image are their edges, lines and points.

In this study we use the edge detection technique in gray level of an image. An edge essentially establishes between two distinctly different regions, which means that an edge is the border between two different regions. 
Here we are using edge detection method for image matching. Edge detection methods locate the pixels in the image that correspond to the edges of the objects seen in the image. The result is a binary image with the detected edge pixels. Segmentation based on thresholding can't be used, as because of different colors of vehicles which cannot be segmented through thresholding.

For Image Matching, in edge detection of matching two images were paired, one reference and other real time acquired image. Then compare then edges of real time images with the reference images in order to get the percentage of matching.

Which identifies the low, medium and high density levels of traffic on roads? On that basis controller will take the decision of time set of traffic signals.
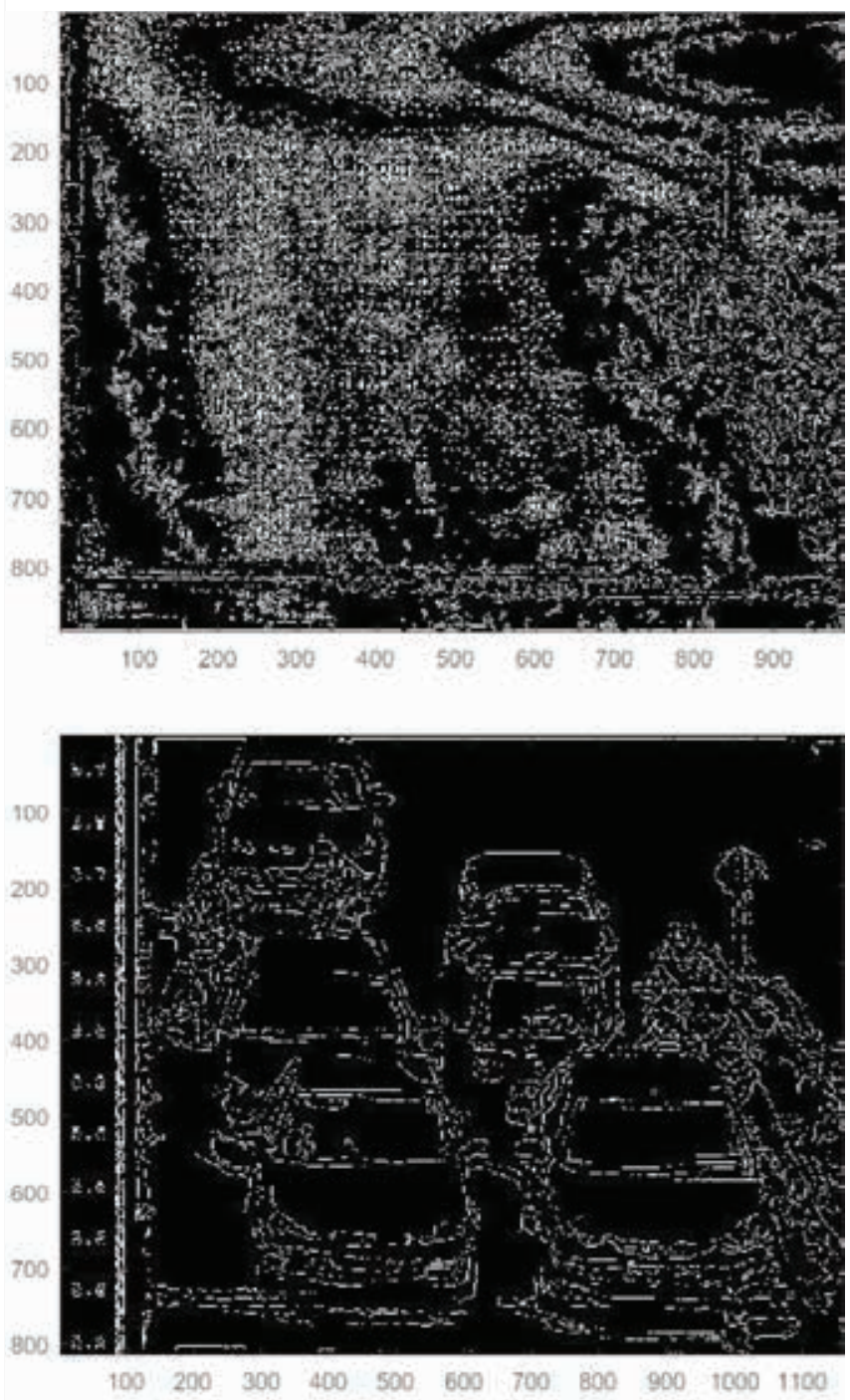

Fig. (8). Output image
The algorithm will then count the number of white and black pixels and then using equation 1,2 and 3 the region will be decided. Demo working of signal is shown below in Table 1.

Table 1. Demo working of signal

\begin{tabular}{|c|c|c|c|c|c|}
\hline Camera & Road 1 & Road 2 & Raod 3 & Road 4 & Duration \\
\hline Innitial Capture & Red & Red & Red & Red & 10 \\
\hline Start with high density & Green & Red & Red & Red & 20 \\
\hline & Yellow & Yellow & Red & Red & 4 \\
\hline Low density & Red & Green & Red & Red & 10 \\
\hline & & Yellow & Yellow & & 4 \\
\hline Low density & Red & Red & Green & Red & 10 \\
\hline & Red & Red & Yellow & Yellow & 4 \\
\hline High density & Red & Red & Red & Green & 20 \\
\hline Emergency at Road 3 & Red & Red & Yellow & Yellow & 4 \\
\hline & Red & Red & Green & Red & till emergency \\
\hline Emergency deared & Yellow & & Yellow & & 4 \\
\hline Normal & Green & Red & Red & Red & 10 \\
\hline
\end{tabular}

\section{CONCLUSION}

The above proposed method of an intelligent traffic control system will definitely plays an important part to reduce the number of traffic congestion problems, road accident and traffic mismanagement related problems. Kindly make note that we are not adding any hardware in the system we are just applying an image processing technique that will upgrade our existing system. The timing of the traffic signal is adjusted with respect to the obtained density. As, night vision cameras are used, that'll help system operate in day and night as well. Such systems would be of great addition to our existing infrastructure as it will not only help us manage the traffic efficiently but also help us reduce the traffic associated cost effectively.

\section{REFERENCES}

[1] D. Ihde, Technology and the Lifeworld. Indiana: Indiana University Press, 1990.

[2] S. H. W. Chuah, P. A. Rauschnabel, N. Krey, B. Nguyen, T. Ramayah, and S. Lade, "Wearable Technologies: The role of Usefulness and Visibility in Smartwatch Adoption," Computers in Human Behavior, vol. 65, pp: 276-284, 2016.

DOI: $10.1016 /$ j.chb.2016.07.047

[3] V. F. Reyna and C. J. Brainerd, "The Importance of Mathematics in Health and Human Judgment: Numeracy, Risk Communication, and Medical Decision Making," Learning and Individual Differences, vol. 17, no. 2, pp: 147-159, 2007.

DOI: $10.1016 /$ j.lindif.2007.03.010 
[4] R. E. Lucas Jr., "On the Mechanics of Economic Development," Journal of Monetary Economics, vol. 22, no. 1, pp: 3-42, 1988.

DOI: $10.1016 / 0304-3932(88) 90168-7$

[5] H. Lund, "Renewable Energy Strategies for Sustainable Development," Energy, vol. 32, no. 6, pp: 912-919, 2007.

DOI: $10.1016 /$ j.energy.2006.10.017

[6] V. Jain, A. Sharma, and L. Subramanian, "Road Traffic Congestion in the Developing World," In Proceedings of the $2^{\text {nd }} A C M$ Symposium on Computing for Development (ACM DEV'12), 2012.

DOI: $10.1145 / 2160601.2160616$

[7] M. Sweet, "Does Traffic Congestion Slow the Economy?," Journal of Planning Literature, vol. 26, no. 4, pp: 391-404, 2011. DOI: $10.1177 / 0885412211409754$

[8] A. de Palma and R. Lindsey, "Traffic Congestion Pricing Methodologies and Technologies," Transportation Research Part C: Emerging Technologies, vol. 19, no. 6, pp: 1377-1399, 2011. DOI: $10.1016 / j . \operatorname{trc} .2011 .02 .010$

[9] G. Cookson and B. Pishue. (2017). INRIX Global Traffic Scorecard [Online]. Available: http://inrix.com/scorecard/

[10] "The Millenium Development Goals Report 2014," Uni. Nat., 2014.
[11] C. Cai, C. K. Wong, and B. G. Heydecker, "Adaptive Traffic Signal Control Using Approximate Dynamic Programming," Transportation Research Part C: Emerging Technologies, vol. 17, no. 5, pp: 456-474 2009.

DOI: $10.1016 / j$.trc.2009.04.005

[12] B. Van Arem, C. J. G. Van Driel, and R. Visser, "The Impact of Cooperative Adaptive Cruise Control on Traffic-Flow Characteristics," IEEE Transactions on Intelligent Transportation Systems, vol. 7, no. 4, pp: 429-436, 2006.

DOI: $10.1109 /$ TITS.2006.884615

[13] D. McKenney and T. White, "Distributed and Adaptive Traffic Signal Control Within a Realistic Traffic Simulation," Engineering Applications of Artificial Intelligence, vol. 26, no. 1, pp: 574-583, 2013.

DOI: 10.1016/j.engappai.2012.04.008

[14] C. R. Lindsey and E. T. Verhoef, "Traffic Congestion and Congestion Pricing," in Handbook of Transport Systems and Traffic Control (Handbooks in Transport.,), D. A. Hensher and K. J. Button, Eds. Eme. Ins., 2001, pp: 77-105.

[15] G. Padmavathi, D. Shanmugapriya, and M. Kalaivani, "A Study on Vehicle Detection and Tracking Using Wireless Sensor Networks," Wireless Sensor Network, vol. 02, no. 02, pp: 173-185, 2010.

DOI: $10.4236 /$ wsn. 2010.22023

(C) Author(s) 2017. CC Attribution 4.0 License. (http://creativecommons.org/licenses/by-nc/4.0/)

This article is licensed under the terms of the Creative Commons Attribution Non-Commercial License which permits unrestricted, non-commercial use, distribution and reproduction in any medium, provided the work is properly cited. 